\title{
Herb Induced Aconite Poisoning and Bidirectional Ventricular Tachycardia
}

\author{
Kasinadhuni G, Vijayvergiya R, Krishnappa D, Kanaba K, Yegurla J \\ and Bhalla $\mathrm{A}^{*}$ \\ Department of Cardiology and Internal Medicine, Post Graduate Institute of Medical \\ Education \& Research, India
}

\section{Case Report \\ Volume 4 Issue 3}

Received Date: July 15, 2019

Published Date: August 18, 2019

DOI: $10.23880 /$ act- 16000161

*Corresponding author: Ashish Bhalla, Department Internal Medicine, Post Graduate Institute of Medical Education \& Research, Sector 12, Chandigarh - 160 012, India, Tel: +91 172 2756536, 9417023973; Fax: +91 172 2744401; Email bhalla.chd@gmail.com

\section{Abstract}

Aconite is a potent cardiotoxic compound and the cardio toxicity gets manifested by hypotension, ventricular tachycardia, and even cardiac death. Herbal medicines may contain aconite as an active gradient, and ingestion may result in rapid clinical deterioration. We hereby report a young lady, who presented with hemodynamically unstable bidirectional ventricular tachycardia following ingestion of an aconite containing herbal medicine. She was successfully managed with vasopressors and anti-arrhythmic drugs. This case emphasises the need for high clinical suspicion of poisoning in patient presenting with cardiac arrhythmias especially following herbal medicine ingestion. In the absence of a definitive antidote, good supportive care remains the key to successful management.

Keywords: Mitha Telia; Herbal Medicine; Aconite Poisoning; Bidirectional Ventricular Tachycardia; Aconitine

\section{Introduction}

Traditional herbal medicines are frequently used either alone or in combination with allopathic medications for various illnesses in South Asian countries. Some of these herbal preparations can be cardio-toxic leading to arrhythmias, circulatory collapse, and even death. Very frequently these herbal preparations are an amalgam of various active ingredients. We hereby report a case of a young lady who had hemodynamically unstable ventricular tachycardia, following ingestion of Mitha Telia, an aconite containing herbal medicine.

\section{Case Report}

A 32-years old female presented in the emergency room with acute onset abdominal pain, recurrent vomiting, diaphoresis, chest heaviness, and palpitations, soon after ingestion of an herbal medicine named "Mitha Telia" for the renal stone disease. On examination, she had the blood pressure of $80 / 60 \mathrm{mmHg}$, irregular pulse of $82 /$ minute with pulse deficit of $8 /$ minute, and systemic oxygen saturation of $96 \%$ at room air. Systemic examination was unremarkable. Electrocardiogram (ECG) showed frequent ventricular ectopics (VPC) and bidirectional ventricular tachycardia (VT) (Figure 1). Routine biochemistry including cardiac biomarkers such as CK-MB and troponin-I were negative. Two-dimensional echocardiography revealed left ventricular ejection fraction of $60 \%$, no regional wall motion abnormality, no mitral regurgitation. She was started on the intravenous infusion of fluids, noradrenaline, and amiodarone to improve the blood pressure and ventricular tachycardia, in the coronary care unit. She gradually improved in the 
next 48-hours. Her ECG reverted back to normal sinus rhythm and without any VPC or VT. She could be discharged on day- 5 on oral amiodarone therapy, which was discontinued after 2-weeks of the asymptomatic follow-up period. Next 6-months of her follow-up were uneventful. At 3 months follow-up, her EKG and echocardiography was normal. The ingested herbal medicine named "Mitha Telia" was found to have an active ingredient- aconitine (Figures $1 \& 2$ ) [1].

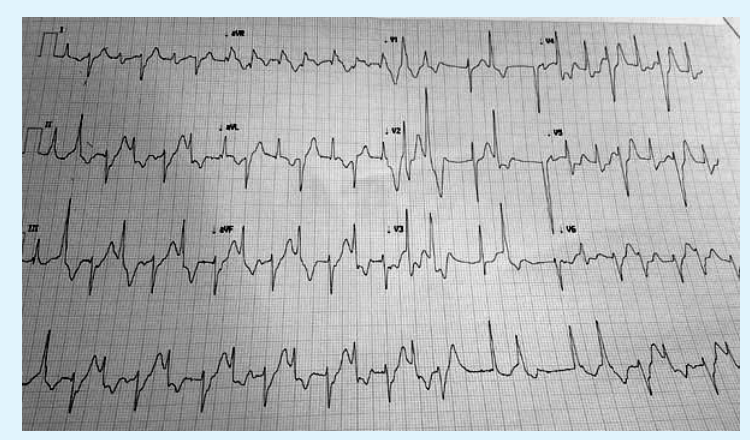

Figure 1: Electrocardiogram showing bidirectional ventricular tachycardia following aconite poisoning.

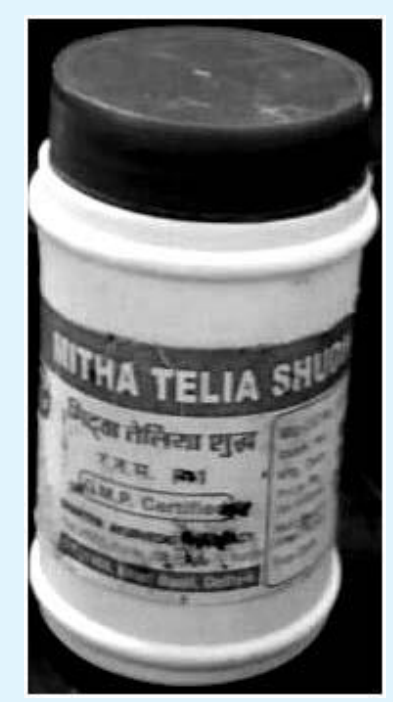

Figure 2: Herbal medicine "Mitha Telia" container. It's active ingredient is aconitine.

\section{Discussion}

Few of the cardiotoxic herbs such as Aconitum ferox, Areca catechu (betel nut), Thevetia nerifolia (yellow oleander) and Cleistanus collinus contains cardiotoxins as aconitine, cardenolides etc [2]. These cardiotoxins can cause life-threatening cardiac arrhythmias, cardiogenic shock and even death. Aconitine is used for pain relief, reflux esophageal disease, and certain neurological disorders [3]. Toxic dose of pure aconite root can be as small as 2-3 mg [4]. As there is a narrow therapeutic range, toxicity may result in cardiovascular mortality, which varies from 5 - $15 \%$ [5,6]. Major metabolic aconite alkaloids are aconitine, mesaconitine and hypaconitine [7]. These toxins are water soluble and excreted in urine by first-order kinetics [5]. Aconitine induced arrhythmias are secondary to increased trigger activity caused by the increase in late $\mathrm{I}_{\mathrm{Na}}$ current. There is a prolonged $\mathrm{Na}^{+}$influx causing increase in cardiac contractility [3,4]. Arrhythmias such as supra-ventricular tachycardia, frequents VPCs, non-sustained VT, polymorphic and bidirectional VT, torsades de pointes, ventricular fibrillation and bradyarrhythmia including conduction blocks had been reported [5,8]. Bidirectional VT as was present in index case is less common but is characteristic of aconite poisoning [9-11]. A high clinical suspicion should be there for aconite poisoning in a case of bidirectional VT with intake of certain herbal medicines. These VTs respond well with $\mathrm{Na}^{+}$channel blocking drugs like flecainide and amiodarone $[4,5]$. The index case responded well with amiodarone. The hypotensive effect is also secondary to toxic central action on the ventromedial nucleus of the hypothalamus as was present in the index case $[3,4]$. Aconitine induced increase in acetylcholine release from post-ganglionic nerves results into increase gastrointestinal motility and clinically manifested as nausea, vomiting, and crampy abdominal pain [3]. Management of aconite poisoning includes vasopressors and fluid resuscitation for hypotension, anti-arrhythmic drugs like flecainide and amiodarone for VT, and other supportive treatment $[3,4]$. Cardiopulmonary bypass and left ventricular assist device for refractory hypotension, and charcoal hemoperfusion for alkaloid toxins in refractory arrhythmias had also been reported in certain cases $[5,6,12]$.

In conclusion, we hereby report a young healthy lady, who presented with hemodynamically unstable bidirectional ventricular tachycardia following ingestion of aconite containing herbal medicine. She was successfully managed with vasopressors, anti-arrhythmic drugs, and supportive treatment.

\section{References}

1. Sood SK, Thakur R (2015) Herbal resources of India and Nepal. Scientific Publishers, pp: 516 
2. Dwivedi S, Aggarwal A, Sharma V (2011) Cardiotoxicity from "safe" herbomineral formulations. Trop Doct 41(2): 113-115.

3. Chan TYK (2009) Aconite poisoning. Clin Toxicol Phila Pa 47(4): 279-285.

4. Karturi SP, Gudmundsson H, Akhtar M, Jahangir A, Choudhuri I (2016) Spectrum of cardiac manifestations from aconitine poisoning. Hear Case Rep 2(5): 415-420.

5. Coulson JM, Caparrotta TM, Thompson JP (2017) The management of ventricular dysrhythmia in aconite poisoning. Clin Toxicol Phila Pa 55(5): 313-321.

6. Lin CC, Chan TYK, Deng JF (2004) Clinical features and management of herb-induced aconitine poisoning. Ann Emerg Med 43(5): 574-579.

7. Imazio M, Belli R, Pomari F, Cecchi E, Chinaglia A, et al. (2000) Malignant ventricular arrhythmias due to Aconitum napellus seeds. Circulation 102(23): 29072908.
8. Lowe L, Matteucci MJ, Schneir AB (205) Herbal Aconite Tea and Refractory Ventricular Tachycardia. N Engl J Med 353: 1532-1532.

9. Smith SW, Shah RR, Hunt JL, Herzog CA (2004) Bidirectional ventricular tachycardia resulting from herbal aconite poisoning. Ann Emerg Med 45(1): 100101.

10. Chakraborty P, Kaul B, Mandal K, Isser HS, Bansal S, et al. (2015) Bidirectional ventricular tachycardia of unusual etiology. Indian Pacing Electrophysiol J 15(6): 296-299.

11. Tai YT, Lau CP, But PP, Fong PC, Li JP (1992) Bidirectional tachycardia induced by herbal aconite poisoning. Pacing Clin Electrophysiol PACE 15(5): 831-839.

12. Fitzpatrick AJ, Crawford M, Allan RM, Wolfenden $\mathrm{H}$ (1994) Aconite poisoning managed with a ventricular assist device. Anaesth Intensive Care 22(6): 714-717. 